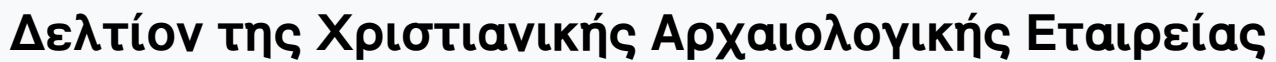

Tó 22 (2001)

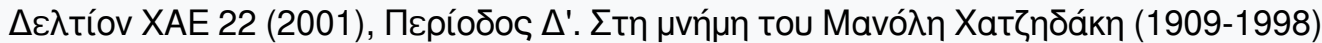

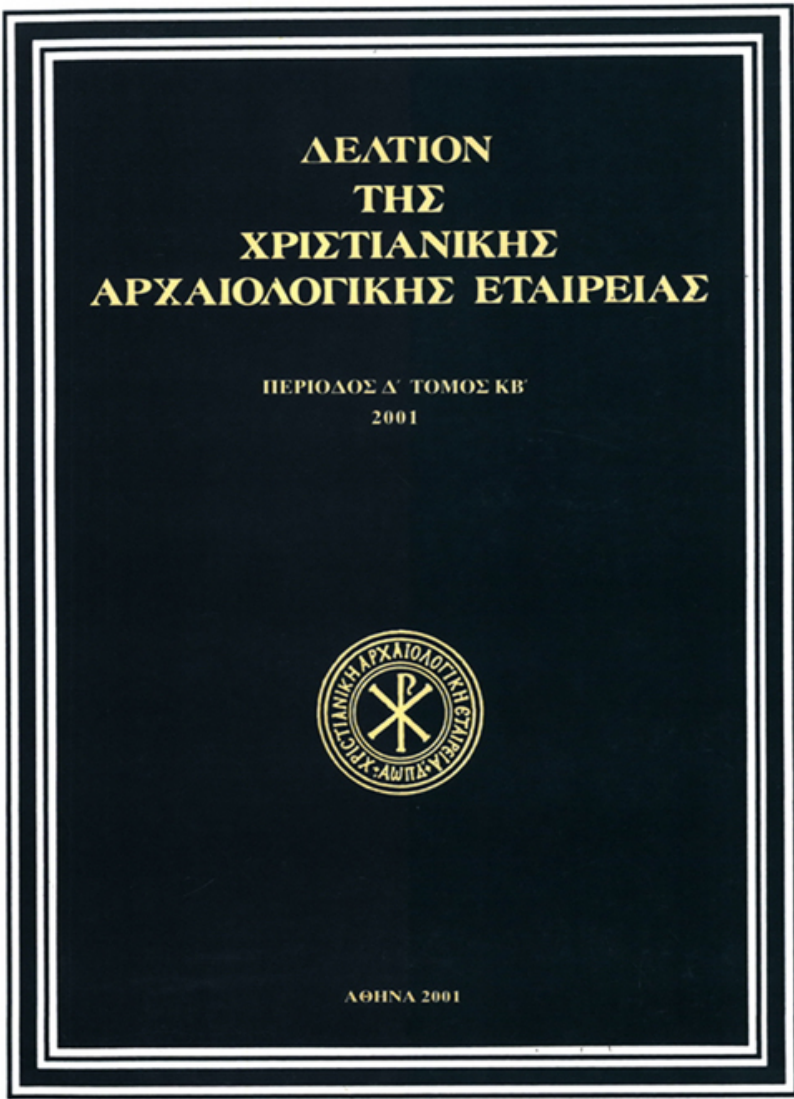

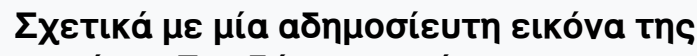

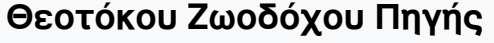

Nicole THIERRY

doi: $10.12681 /$ dchae.316

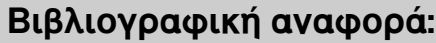

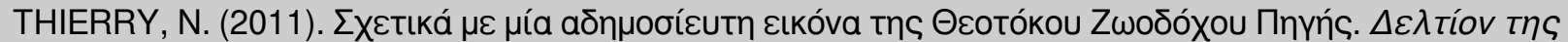

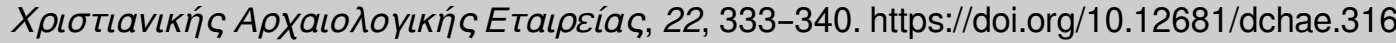




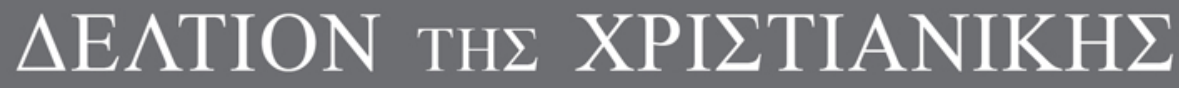 APXAIO $\Lambda$ ОГКН $\Sigma$ ETAIPEIA $\Sigma$}

Sur une icône inédite de la Vierge Source de vie

Nicole THIERRY

Tómos KB' (2001) • $\Sigma \varepsilon \lambda . ~ 333-340$

A@HNA 2001 


\section{Nicole Thierry}

\section{SUR UNE ICÔNE INÉDITE DE LA VIERGE SOURCE DE VIE}

L' icône de la Zoodochos Pege que nous présentons (Fig. 1) s'inscrit dans une série dont les exemples les plus représentatifs sont l'icône de la collection Abou-Adal, datant du XVIIIe siècle, et celle du Musée Byzantin d'Athènes, de $1793^{1}$; elle s'en distingue cependant par la place importante faite à la Théotokos dans la phiale.

Cette icône inédite était dans le commerce à Stamboul en 1968. Elle est tout d'une pièce; la peinture s'étend sur une surface en réserve, entourée d'une importante décoration architecturale formant cadre. L'œuvre est en bois tendre et vermoulu qui a nécessité un traitement. Au revers, le bois est brut. L'ensemble mesure $38 \mathrm{~cm}$ de large sur 53 de haut, la peinture n'occupant que $18 \mathrm{~cm}$ sur 28 .

Le bois sculpté et doré est d'un style dérivé du baroque occidental; il met en valeur la Théotokos (MP $\Theta Y$ ) de la peinture. Le fronton arqué représente deux anges tenant au-dessus d'elle une haute couronne globulaire, tandis que la colombe du Saint Esprit descend verticalement sur sa tête. En bas, un ange, ou plutôt sa tête entre les ailes déployées, semble soutenir l'image. Le reste du décor est plus profane : latéralement des colonnes détachées du fond et surmontées d'un chapiteau composite (ionique surmonté d'une haute feuille d'acanthe), soutiennent la corniche où des feuillages abritent deux grands oiseaux qui se retournent, le cou tendu et les ailes ouvertes (mi-aigles, mi-échassiers, oiseaux mythiques plus que réels). L'unité de l'ensemble est assuré par des rinceaux fleuris, amples feuillages se détachant en or sur des branchages brun foncé en arrière-plan ${ }^{2}$. La composition architecturale se rapproche de celle de l'icône Abou-Adal (Fig. 2), mais elle est d'un art baroque à la fois plus sophistiqué et plus rustique avec l'ajoût original de la colombe. De plus, le cadre est sculpté en assez haut-relief, ce qui évoque singulièrement un autel d'église et sa valeur votive.

On sait que la source miraculeuse entraîna la fondation d'un riche monastère par Justinien ${ }^{3}$. Le sanctuaire devint un des plus fréquentés et célèbres de Constantinople en raison du pouvoir guérisseur de ses eaux. Il subit diverses réparations et embellissements sous Irène (vers 790) puis Basile Ier après 869 , Léon VI et Romain Lécapène. Les empereurs y suivaient les offices du jeudi de l'Ascension et utilisaient comme lieu de réception un petit château voisin construit par Basile Ier ${ }^{4}$. L'occupation latine aurait été marquée par la perte du pouvoir thaumaturge de la source. Le monastère restauré au tout début du XIVe siècle connut alors son apogée. Il semble avoir été détruit et abandonné après la chute de Constantinople, mais les pèlerinages se perpétuèrent. En 1727, une chapelle fut bâtie que les Janissaires détruisirent, comblant la source, en 1821. Enfin en 1835 le patriarche consacra une nouvelle église qu'un firman avait autorisée, la source continuant à attirer les fidèles ${ }^{5}$. Aujourd'hui encore,
1. M. Chatzidakis, Les icônes byzantines et postbyzantines, Icônes grecques, melkites et russes, Collection privée du Liban, Genève 1993, $\mathrm{n}^{\circ}$ 46, p. 154-155 (pl. en couleurs) ; encore dans Lumières de l'Orient chrétien. Icônes de la collection Abou-Adal, Genève 1997, nº 49, p. 122 (renvoi à deux icônes, l'une au Musée d'Art et d'Histoire de Genève, l'autre au Musée Byzantin d'Athènes). Encore M. Chatzidakis, Icônes de SaintGeorges des Grecs et de la Collection d l'Institut de Venise, Venise 1962, p. 171 (trois peintures apparentées, cadres non décrits, bibliographie). L'icône d'Athènes est cotée BM 1776 / T. 1323, cf. D.I. Pallas, The Theotokos-Zoodochos Pighi, Iconographical Analysis and History of the Subject, $A \Delta 26$ (1971), A, p. 201-224 (résumé anglais, 322-324), p. 219-221, pl. 53, 54 .
2. Mme K. Platchkov, expert à Genève, nous a signalé l'existence de cadres comparables du XVIIIè siècle, originaires d'Asie Mineure, ce dont nous lui sommes reconnaissante.

3. R. Janin, Eglises et monastères de Constantinople, Paris 1953, p. 232237 (importante bibliographie), d'après Procope, De Aedif., I, 3, 6-8. La première église serait du Ve siècle, fondée par Léon Ier (457-474), d'après la légende transmise par Nicéphore Calliste au début du XIVe siècle ; J. Ebersolt, Sanctuaires de Byzance, Paris 1921, p. 61-66.

4. Constantin VII Porphyrogénète, Le livre des cérémonies, I, 27 (18), éd. A. Vogt, Paris 1935, p. 101-105 et les Commentaires, p. 87, 129-130; Anne Comnène, Alexiade, I, 16, éd. B. Leib, Paris 1937, p. 5.

5. Janin, op.cit., p. 235 ; Ebersolt, op.cit., p. 62-64. 


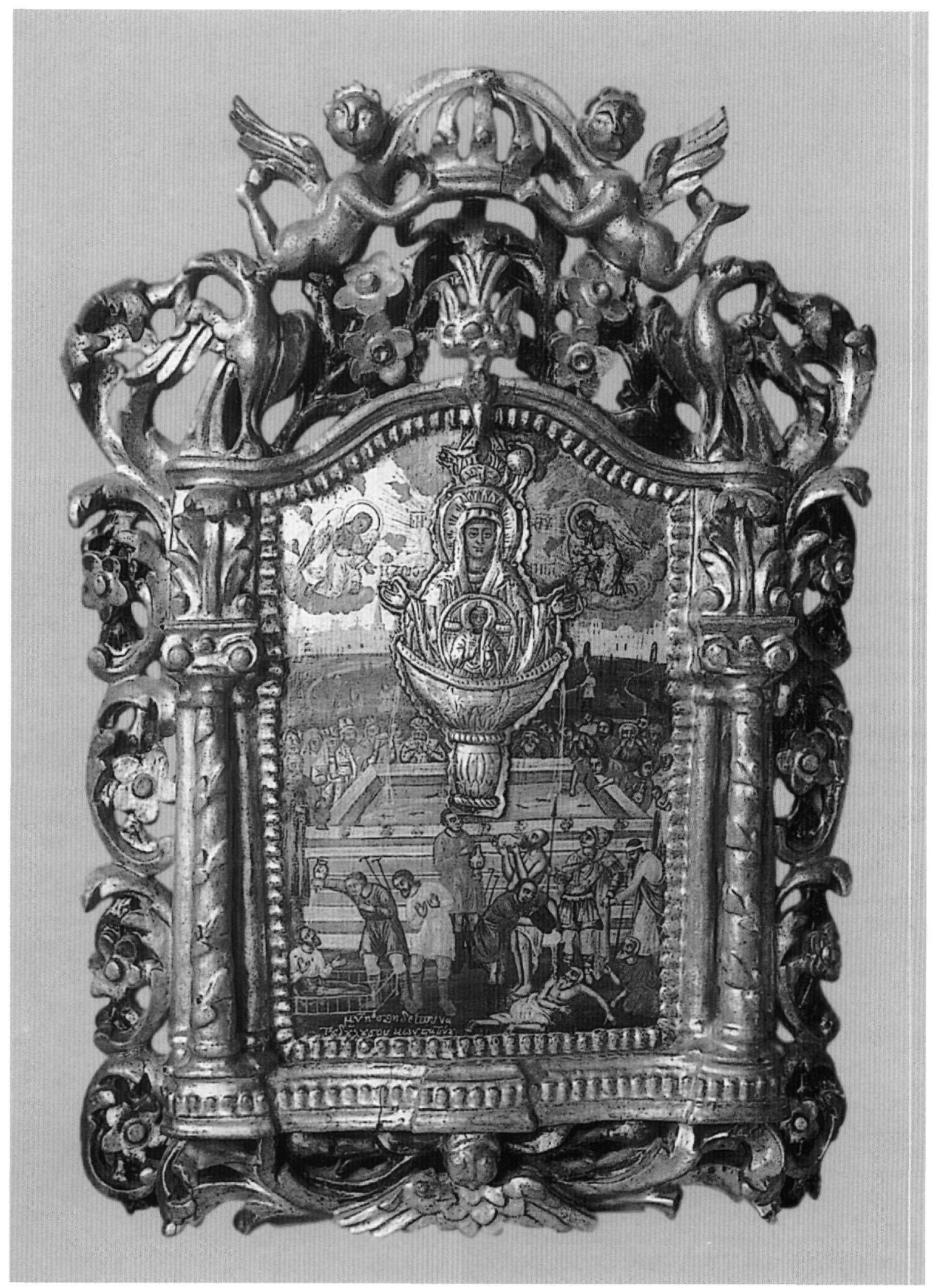

Fig. 1. Icône de la Vierge Source de vie. 
un cimetière où reposent des patriarches et un modeste monastère habité par quelques nonnes restent un lieu de pèlerinage traditionnel des visiteurs grecs; une citerne souterraine où nagent des poissons rouges correspond à l'aghiasma de jadis et les icônes de la Zoodochos Pege conservent un pouvoir protecteur des malades ${ }^{6}$. La Vierge de la fontaine de vie devint si populaire que les églises et monastères mis sous ce vocable se multiplièrent dans le monde postbyzantin, notamment au Proche Orient et les icônes la reproduisant connurent un grand succès populaire ${ }^{7}$.

Après le hiatus de l'Empire latin, les moines grecs réoccupèrent donc le monastère dans les dernières années du XIIIe siècle et s'efforcèrent de lui rendre son prestige passé, avec succés. Les pèlerins recommencèrent à affluer, le pouvoir guérisseur de l'eau sainte étant de nouveau attesté (notamment pour Andronic III en 1330). Le sanctuaire connut un renouveau de gloire dans les fastes impériaux comme dans la fréquentation des fidèles, ceci jusqu'à la fin de l'Empire.

Une nouvelle et récente étude des épigrammes de Manuel Philes consacrés à la Zoodochos Pege met en évidence cette renaissance de l'église et de la fréquentation de la source ${ }^{8}$, cette dénomination s'instaurant alors que jusqu'ici le sanctuaire était dit de la Théotokos tes Peges, ou Pege 9 .

Les épigrammes, plus ou moins longs et dédiés à la Théotokos, étaient destinés à être écrits sur des icônes offertes à l'église, ou sur des objets liturgiques, voire sur des pièces de réparation (comme un réservoir, don d'un moine Hilarion $)^{10}$. Les donations accompagnaient généralement la demande d'une guérison ou des remerciements pour celle-ci. Ce dernier type d'ex-voto faisait parfois mention de miracles précis : guérison d'une main paralysée (?), d'hémorragies utérines, d'hydropisie, de céphalées, de maladie infantile, d'une lèpre ${ }^{11}$. Malheureusement, les icônes du XIVe siècle ont disparu et il est difficile de savoir comment les peintres avaient traité le sujet. Il est vraisemblable cependant que les

6. Pour l'état du monastère à la fin du siècle dernier, cf. la fig. de la p. 151 dans G. Schlumberger, Un empereur byzantin au Xe siècle, Nicéphore Phocas, Paris 1890. Description sommaire de l'aghiasma de Balıklı dans le Guide Bleu de 1958, p. 158. Nous remercions ici Brigitte Pitarakis qui nous a renseignée sur la fréquentation actuelle et sur l'usage encore récent d'accrocher ce type d'icône au-dessus du lit des malades.

7. Janin, op.cit., p. 247. M. Mahmoud Zibawi, spécialiste du Christianisme oriental, que nous remercions ici, nous a signalé le bon nombre d'images de Zoodochos Pege au Liban et en Syrie, icônes du XIXe siècle de qualité médiocre et très narratives, une série provenant d'un atelier de Jérusalem.

8. Alice-Mary Talbot, Epigrams of Manuel Philes on the Theotokos tes

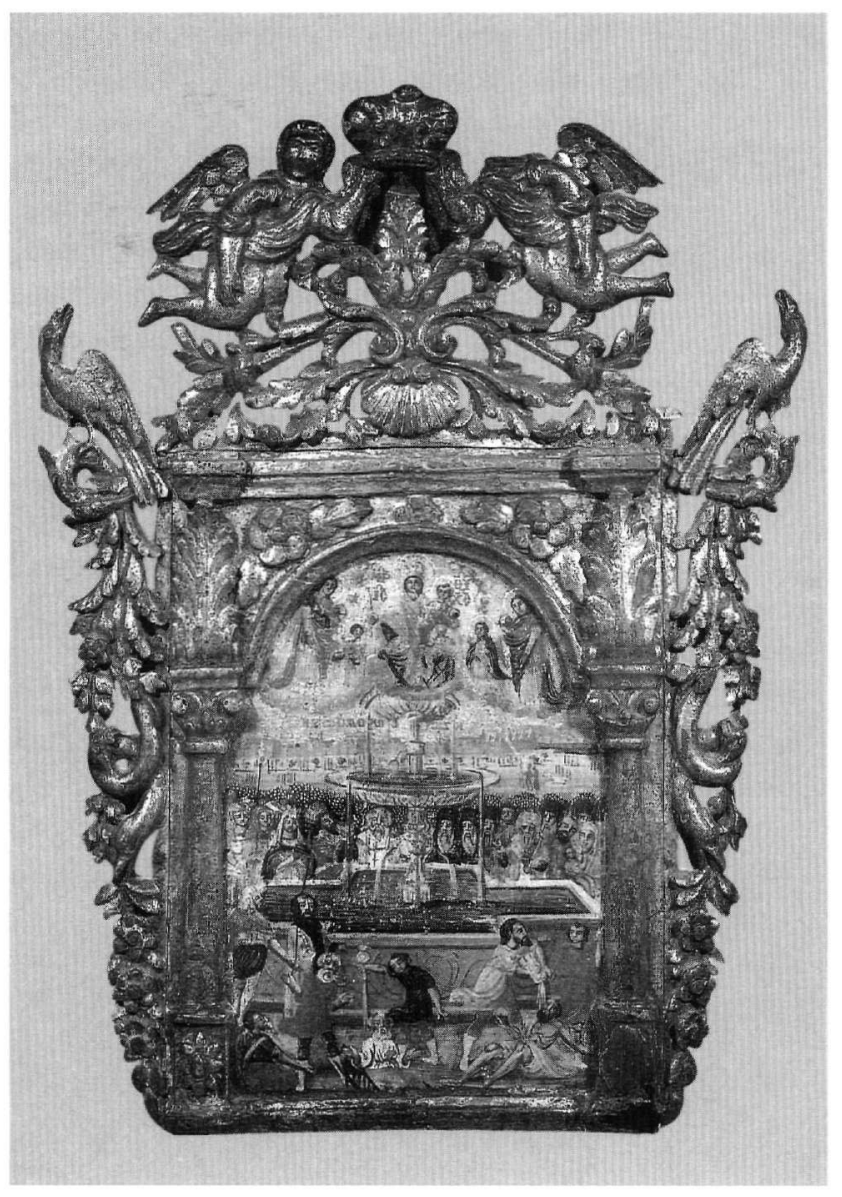

Fig. 2. Icône Abou-Adal (d'après Icônes, Genève 1993, p. 155).

icônes du XVIIIe siècle qui nous sont parvenues en ont gardé quelque chose, développant la narration pittoresque des diverses guérisons aux dépens de l'image théophanique de la Théotokos, cependant la vraie source des miracles ${ }^{12}$.

Peges and its Art, DOP 48 (1994), p. 135-165.

9. Constantin VII, op.cit. (n. 4), p. 102. L'Epithète avait été appliqué pour la Vierge elle-même, notamment au IXe siècle par Joseph l'Hymnographe mais n'avait pas été étendu au sanctuaire, appelé seulement Pege, Talbot, op.cit., p. 136 et n. 7 et 8 .

10. Talbot, op.cit., liste p. 142.

11. Talbot, op.cit., épigr. $\mathrm{n}^{\mathrm{os}} 8,9,11,12,15$.

12. Sur place, au XIVe siècle, la mosaïque de la coupole couvrant la fontaine représentait la Vierge dont l'image se reflétait dans l'eau. Mais Nicéphore Calliste glorifiait également le pouvoir de la source, recensant soixante-trois guérisons miraculeuses dont quinze de son temps, Janin, op.cit., p. 236, n. 3. 


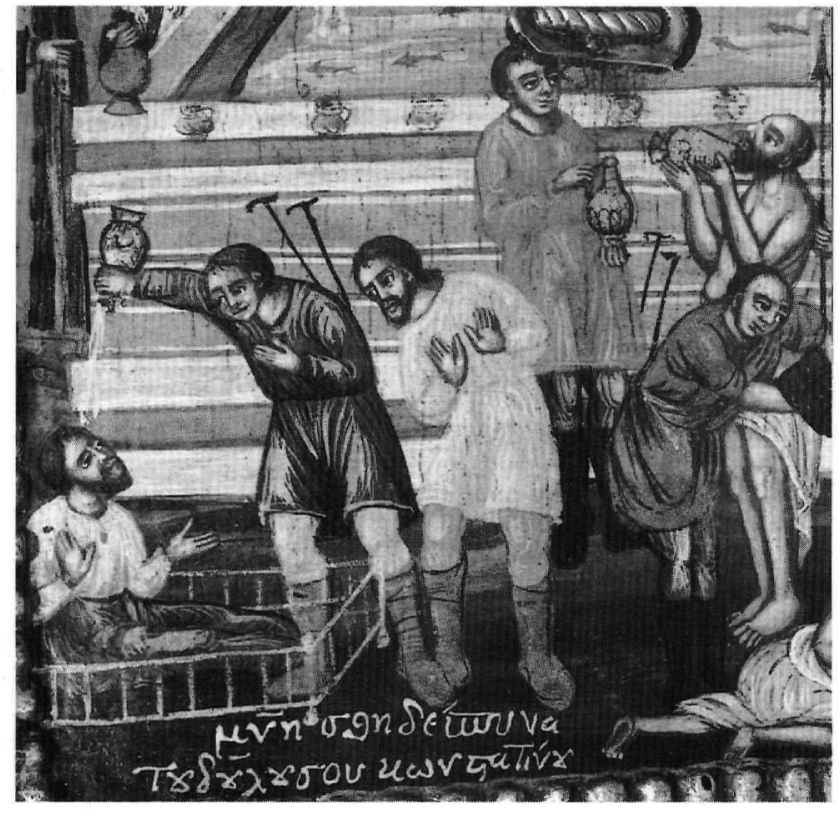

Fig. 3. Angle inférieur gauche de l'icône.

Notre icône porte le nom du commanditaire (Fig. 3), un certain Constantin dont la prière est bien conservée, en bas et à

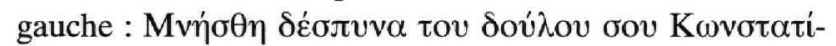
vou, Souveraine, souviens-toi de ton serviteur Constantin ${ }^{13}$.

Elle fut peut-être peinte pour accompagner un vœu qui n'est pas précisé et il est vraisemblable que ce fût une icône d'usage domestique destinée à protéger Constantin de la maladie ${ }^{14}$.

Les moines de Pege proposaient sans doute aux pèlerins de ces icônes à vertu prophylactique, associant l'image de la Théotokos et le spectacle de l'activité curative de la source. Dans le cas présent, la foule variée des curistes et la représentation de quelques célèbres guérisons ont constitué une composition qui correspond à une typologie particulière que nous retrouvons sur l'icône du Musée Byzantin d'Athènes (Fig. 4), et en partie sur un triptyque de 1798 rapportée de Cappadoce et, quoique appauvrie, sur une chalcographie de Nicolas de Chio datée de $1812^{15}$.

13. Lu par Georges Kiourtzian, ce dont nous le remercions (cursive assez élégante, avec ligatures).

14. Sur les icônes comme images pieuses de la maison, Th.F. Mathews, Byzantium from Antiquity to the Renaissance, New York 1998, 43-52, fig. 28.

15. Pallas, op.cit., p. 219-221, pl. 53-55.
En revanche, la nôtre se différencie par sa typologie de la Théotokos de la Source qui est du type traditionnel au XIVe siècle; de plus, elle est surmontée de la colombe et de Dieu le Père, éléments de l'iconographie baroque occidentale (Fig. 1). La Vierge est du type Blachernitissa, en buste dans une phiale, les bras levés en orante ; l'enfant en médaillon devant elle, tient le rouleau et bénit. L'image est conforme à la description de Manuel Philes dans son épigramme sur l'icône de Pege qui porte la source d'immortalité en son sein ${ }^{16}$. Un revêtement d'argent a été ultérieurement fixé pour enrichir le sujet, il empiète latéralement sur une partie de l'inscription $\mathrm{H} \mathrm{Z \Omega O} \triangle(\mathrm{OXOC)}$ ПНГН (Source qui reçoit la vie). La représentation, avec les anges Michel et Gabriel en prière latéralement, est traditionnelle depuis les images anciennes, celles des peintures de l'Afendiko (vers 1308) et des SaintsThéodores à Mistra $(c a 1400)^{17}$.

Notre peintre a ajouté un registre céleste avec les deux autres éléments de la Trinité, la colombe et Dieu le Père au centre des nuages, figurés à petite échelle. Ce thème de glorification de la Vierge a été reproduit sur la plaque d'argent : au dessus de la tête couronnée, figurent la colombe aux ailes déployées, difficile à distinguer sous le buste du Père tenant le globe et bénissant. La pièce métallique laisse en réserve les visages de l'Enfant, de la Vierge et de Dieu, ce dernier très petit alors que s'impose celui de la Vierge, d'une belle plastique et au regard appuyé.

Le métal recouvre aussi la phiale et la colonne qui se dresse dans le bassin carré. Un filet ondulé descend le long du support, figurant l'eau qui coule de la source sainte, alors que deux jets d'eau sont peints latéralement.

Malgré la présence (d'ailleurs réduite) du registre supérieur, l'importance de l'image centrale de la Théotokos est remarquable et distingue cette composition de celles des icônes du Musée Byzantin d'Athènes et de la collection d'Abou-Adal caractérisées par la préciosité de l'ornementation des fontaines à deux étages et la représentation de la Vierge trônant sur les nuages et tenant l'Enfant sur le bras comme l'Odigitria.

L'image primitive de la Blachernitissa dans la phiale s'est également perpétuée jusqu'aux temps modernes et notre

16. Talbot, op.cit., p. 142-143. Ici, l'Enfant regarde légèrement vers la droite alors que la Vierge est parfaitement de face.

17. Suzy Dufrenne, Les programmes iconographiques des églises byzantines de Mistra, Paris 1970, p. 5, 9, 41. Schéma de G. Millet dans Talbot, op.cit., fig. 2 d'après G. Millet, Monuments byzantins de Mistra, Paris 1910, pl. 90 ; Ebersolt, op.cit. (n. 3), fig. 11; Pallas, op.cit., pl. 49. 
icône n'en est qu'un exemple ${ }^{18}$. On peut la considérer comme la forme officielle et elle est d'ailleurs celle que décrit Denys de Fourna ${ }^{19}$. Cette tradition traduit un souci de religiosité qui ramène à l'essentiel, comme pour la composition de l'église Saint-Jean de Mistra, la plus ancienne qui nous soit parvenue montrant le bassin aux guérisons miraculeuses, dans lequel plonge une courte et large colonne soutenant la phiale (Fig. 8).

En bref, le culte de la Vierge équilibre celui des eaux miraculeuses et la piété ne le cède pas au goût du pittoresque. C'est bien d'elle qu'on attend le maintien ou le retour de la santé. Dans le cas présent, l'ornement métallique et la composition supérieure du cadre sculpté renforcent ce sentiment de pouvoir guérisseur de l'image de la Théotokos.

La fonction narrative de l'icône n'a cependant pas été négligée. Le charme du paysage et le tableau animé de la source sont du même ordre que sur les deux icônes déjà citées. L'or $\mathrm{du}$ fond et des accessoires tels que casques ou couronnes, et l'alternance des couleurs vives des vêtements où brillent les étoffes rouges confèrent une grande vivacité à la composition. Apparemment, rien ne rappelle l'architecture originale de l'église décrite par Nicéphore Calliste ${ }^{20}$; on ne retrouve que la situation hors de la ville, dans un lieu plaisant planté de platanes et de cyprès.

En fait, ici, le bassin et la phiale paraissent isolés dans une campagne peu arborée où serpentent des sentiers par lesquels arrivent des malades. A droite (Fig. 5), on remarque un boiteux et un paraplégique agenouillé pour prier, ayant posé devant lui les deux poignées d'appui qui l'aident à se traîner ${ }^{21}$. Au fond du paysage, la barre des murailles blanches de Constantinople se détache sur le fond or. Derrière les créneaux on voit une coupole et deux portes, l'une encadrée par deux tours, l'autre surmontée d'une seule. A gauche (Fig. 6), deux flèches se dressent que nous pensons être celles d'églises latines (?) et une coupole d'église grecque surmontée d'une croix ainsi qu'une porte et une tour.

Le centre de l'activité est la bassin rectangulaire, de pierre bleutée, dans lequel l'eau est agitée par celle qui tombe de la

18. Pallas, op.cit., schéma 3, pl. 50, 51, 55.

19. M. Didron, Manuel d'iconographie chrétienne, traduction du Guide de la peinture, Paris 1845, p. 288-290 (la description des foules et miracles s'accordent à peu près, à la fois aux compositions de l'icône Abou-Adal et de celle du Musée d'Athènes et de la nôtre).

20. Nicéphore Calliste, Hist. eccl. XV, 26 (PG 147, 72-78).

21. Jusqu'au début de notre siècle, on a vu ces infirmes, et les culs-

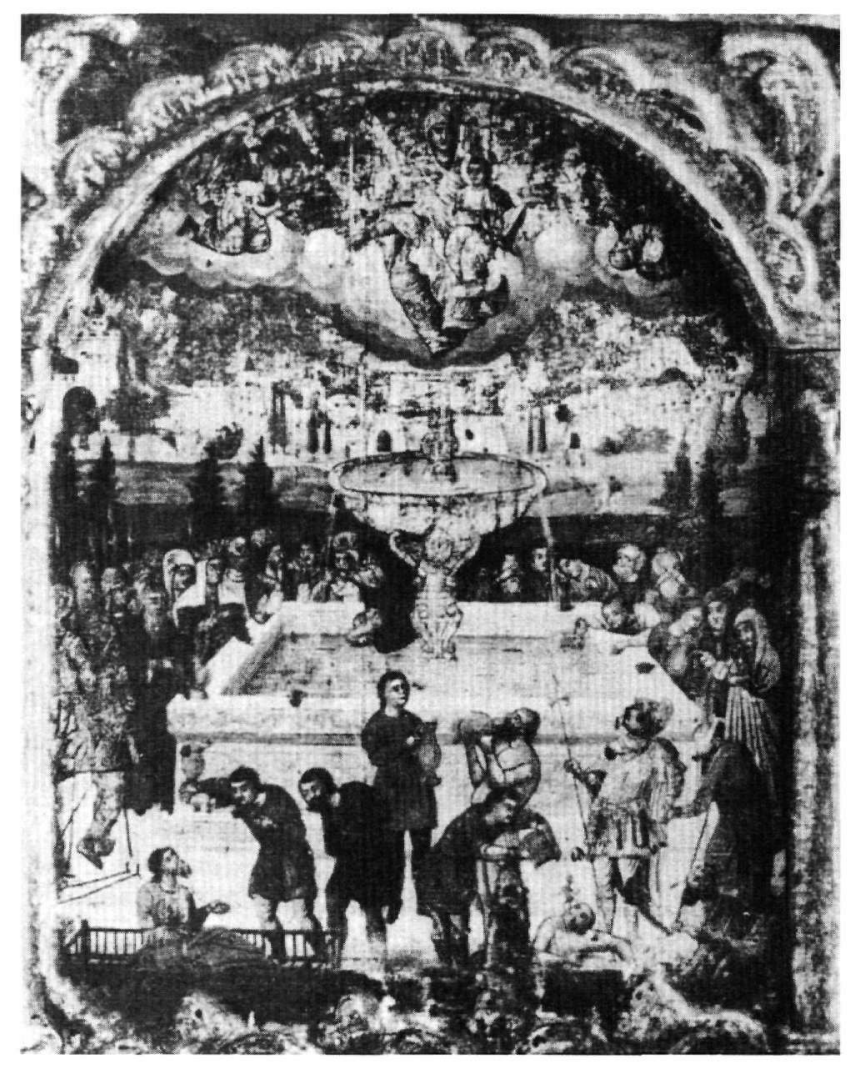

Fig. 4. Icône du Musée Byzantin d'Athènes (d'après Pallas 1971, pl. 53).

phiale et par quelques poissons qu'on pouvait pêcher et manger ; cette nourriture s'ajoutait aux bienfaits de l'eau sacrée. Une lettre de Michel Gabras parlant du séjour de son frère au sanctuaire, décrit comment il obtint une rémission de son mal (l'hydropisie) en priant continuellement et refusant toute nourriture autre que l'eau de la source et un petit poisson $^{22}$.

Des tasses destinées aux malades sont disposées sur le bord antérieur du bassin, et en avant sont appuyées des cannes abandonnées par des infirmes partis guéris (Fig. 3). Les pè-

de-jatte sur leur planche à roulettes, utiliser comme poignées des fers à repasser.

22. Cité par Talbot, op.cit., p. 137-138, n. 14 et 15 ; cette première mention de manducation des poissons est de peu antérieure à la mort de son frère en 1319 ; elle est citée ensuite par un pèlerin russe anonyme en 1390. 


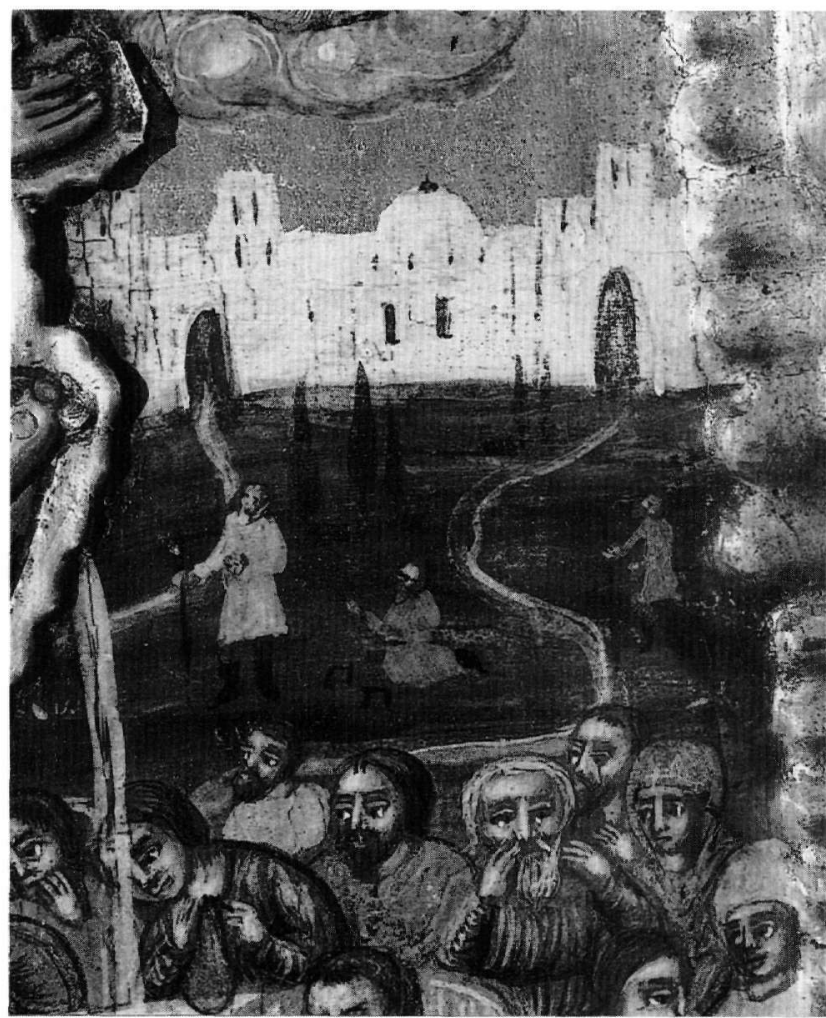

Fig. 5. Moitié droite du paysage.

lerins présents sont divisés en trois groupes : les personnages importants sur le côté gauche, les gens du peuple à droite et au premier plan quelques scènes de guérison (Fig. 1). La nette séparation des puissants de ce monde et du reste de la population indique combien toutes les classes de la société se pressaient à la source miraculeuse, conformément aux témoignages littéraires et aux récits hagiographiques. La composition aussi bien que la plupart des détails reproduisent les sujets de l'icône du Musée Byzantin d'Athènes (Fig. 4) ${ }^{23}$. Dans la foule massée à gauche (Fig. 6), se tiennent au premier rang un patriarche, un empereur couronné suivi d'un militaire casqué et armé d'une hallebarde ; d'autres soldats sont en arrière de l'impératrice et d'une noble dame. Patriarche, empereur et impératrice boivent dans des coupes. Sur le côté, un notable âgé, vêtu d'un manteau rouge bordé de noir, tient un vase et montre la source.

Le groupe de droite est plus animé et les malades sont individualisés (Fig. 7 et 5). Vers le centre, un vieillard mouille ses yeux avec l'eau miraculeuse ; près de lui deux jeunes gens se penchent, l'un tenant un vase qu'il va remplir ; derrière eux deux adultes barbus les séparent d'un vieillard qui lave son visage avec ses mains, tandis qu'un jeune homme se

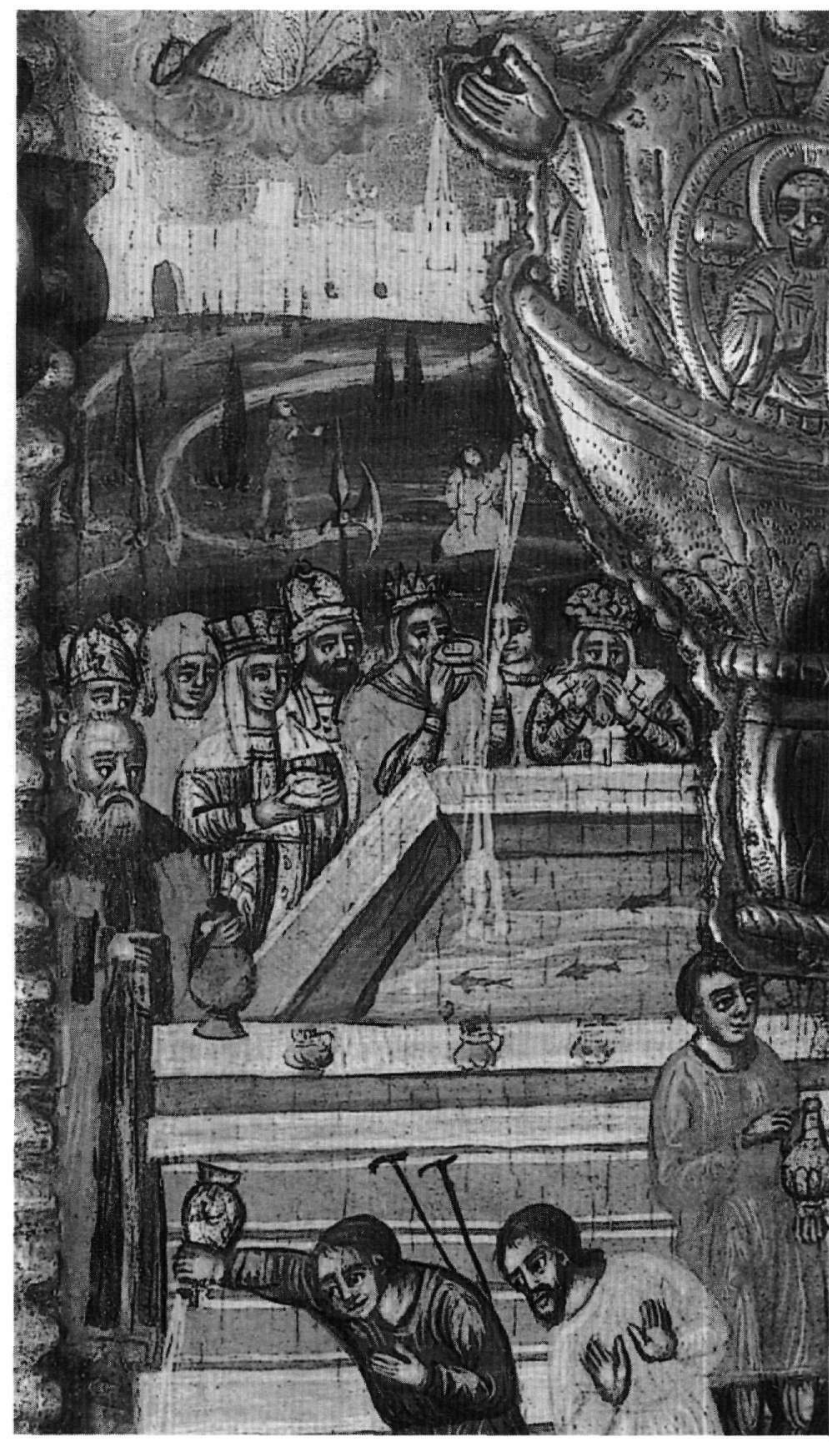

Fig. 6. Côté gauche de l'icône.

penche sur l'eau pour remplir un vase. Enfin, un groupe de trois femmes occupe le premier plan : une jeune servante aux longs cheveux verse l'eau d'une aiguière sur un enfant dénudé que sa mère tend vers la source, tandis qu'en arrière, la troisième assiste à la manœuvre.

23. Pallas, op.cit., pl. 53. La composition des foules de l'icône Abou Nadal et du triptyque d'Athènes est différente, opposant à gauche des personnages de la cour, à droite, des membres du clergé (Chatzidakis, op.cit., n. 1 ; Pallas, op.cit. pl. 55 $\alpha$ ), ce qui correspond au texte de Denys de Fourna, op.cit., p. 289. 
Devant la piscine, figurent quelques guérisons miraculeuses représentées à plus grande échelle que les foules massée en arrière et le long des bords latéraux. A gauche (Fig. 3), un jeune homme verse l'eau d'un vase sur la tête d'un vieillard étendu sur un matelas et qui se redresse, les bras levés et les yeux tournés vers la Théotokos en reconnaissance du miracle. Un témoin, jeune homme barbu, les deux paumes levées, semble s'émerveiller du phénomène. Il s'agit vraisemblablement de la résurrection d'un voyageur de Thessalie mort en cours de route et transporté par des marins qui l'aspergèrent de l'eau salvatrice. Cette scène identifiée par Manolis Chatzidakis sur l'icône Abou-Adal y est mieux définie car le matelas du vieillard paraît posé sur un brancard et non dans cette sorte de lit-cage, ou petit parc à barreaux de bois, moins facile à transporter. Cependant, c'est ce dernier qu'on retrouve ailleurs, notamment sur le triptyque publié par Neuffert ${ }^{24}$ et sur l'icône d'Athènes (Fig. 4).

La guérison d'un possédé fait suite à droite (Fig. 7): un jeune homme verse un seau d'eau sur le vieillard demi-nu étalé sur le sol. Sur l'icône d'Athènes, la petite silhouette noire du démon sort de la bouche du possédé, bien visible sur le fond clair (Fig. 4), alors qu'ici, la forme noire se distingue mal. En arrière, plus près du bassin, on voit l'hydropique, seulement vêtu d'un pagne, buvant à un vase ; face à lui un assistant s'apprête à lui tendre une aiguière pleine (Fig. 7 et 3). L'hydropisie, identifiée ici au gros ventre du malade, était un des maux les plus souvent traités avec succès à Pege, dont les eaux avaient sans doute des propriétés diurétiques ${ }^{25}$.

Enfin, sur le côté droit s'avance un aveugle s'appuyant sur son bâton et guidé par un soldat tenant une pique et vêtu à l'antique : tunique courte et cuirasse dorées, grand manteau et casque (Fig. 7). Le soldat lève les yeux vers la Théotokos, comme si elle s'adressait à lui. La scène illustre assez bien l'épisode légendaire conté par Nicéphore Calliste et qui serait à l'origine d'une première église fondée par Léon Ier (457-474). Alors que, simple soldat, Léon le Thrace arrivait à Constantinople près des marais qui avoisinaient la Porte dorée, un aveugle le pria de lui servir de guide et de lui donner à boire. Pendant que Léon cherchait de l'eau, il entendit une voix qui disait "L'eau est près de toi »... et lui ordonnait de frotter les yeux de l'aveugle avec l'eau du marais, ajoutant qu'elle avait

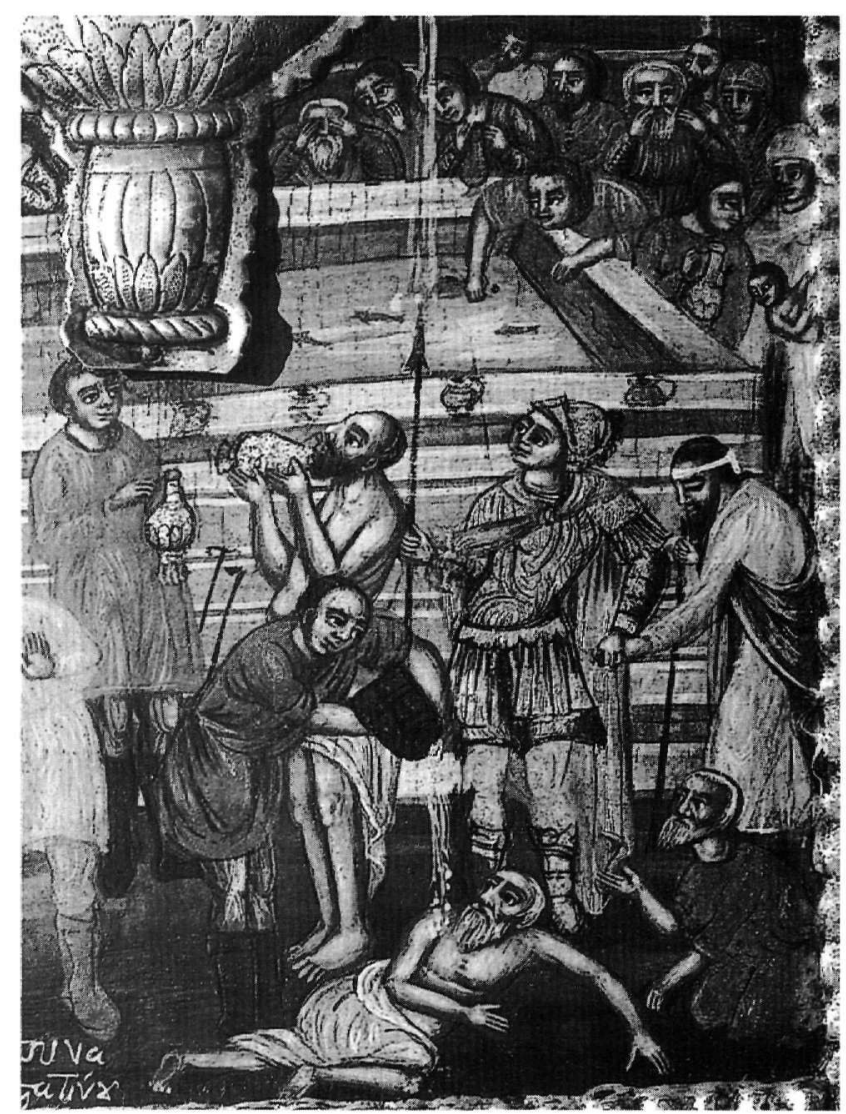

Fig. 7. Partie inférieure droite de l'icône.

choisi ce lieu pour y être honorée et qu'une fois devenu empereur, il devrait bâtir une église où l'on viendrait chercher la guérison. Léon obéit, frotta les yeux de l'aveugle qui recouvrit la vue. Après son accès à l'empire (7 février 457) il aurait construit une église qui aurait précédé celle de Justinien ${ }^{26}$.

Sur l'icône Abou-Adal, l'épisode est évoqué par deux petites silhouettes rouges dans les lointains du paysage ; c'est le soldat armé de la pique qui conduit un personnage courbé 27 . En revanche, la scène figure à l'identique sur l'icône d'Athènes et confirme l'existence d'un modèle commun pour l'ensemble des scènes. Il ne manque même pas le dernier plaignant, dans l'angle inférieur droit, un paraplégique
24. Pallas, op.cit., schéma 3.

25. Talbot, op.cit., p. 137-138, n. 14-15; p. 157, n. 105. En fait, l'hydropisie, caractérisée par une infiltration massive des tissus, a de multiples origines dont certaines pouvaient être sensibles à la composition miné- rale des eaux de Pege.

26. Janin, op.cit. (n. 3), p. 233, d'après $P G$ 147, 72-77B.

27. Chatzidakis, op.cit. (n. 1). 


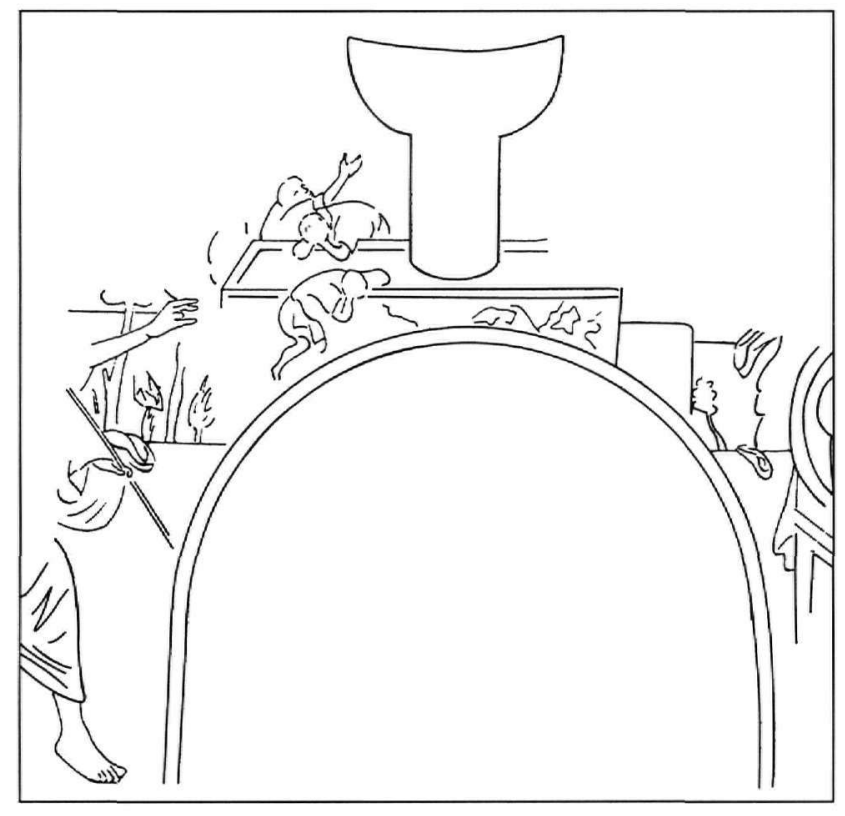

Fig. 8. Schéma d'après la peinture de Saint-Jean de Mistra (d'après Millet 1910, pl. 107).

qui se traîne dans l'attente du miracle qui lui rendra l'usage de ses jambes. Outre le style, plus populaire de la nôtre, on remarque que le tableautin des scènes narratives a été resserré. Sur le côté droit, la femme à l'enfant malade est à demi-visible et à gauche, l'image s'arrête au vieillard en manteau rouge et au ras du dos du ressuscité.

Le parallélisme de ces deux icônes, est un nouveau témoignage d'une production d'images pieuses étroitement liée au monastère de la Zoodochos Pege au cours du XVIIIe siècle et peut-être au XIXe, après la dernière renaissance du sanctuaire. Dans le même atelier, les œuvres pouvaient varier en fonction du peintre, du prototype choisi parmi les représentations de la source, de l'addition d'un détail pieux ou d'un schéma plus ancien de la Vierge Source de vie.

Ici, l'artiste a reproduit le même modèle que celui de l'icône du Musée Byzantin d'Athènes pour la représentation du bassin, des foules qui s'y pressent et des miracles célèbres qui témoignaient de l'antiquité et de la continuité du pouvoir miraculeux de la fontaine. Le paysage des abords de Constantinople est différent et sans doute était-il laissé à l'initiative de l'artiste, ainsi voit-on une autre variante sur l'icône Abou-Adal. Enfin, pour l'image de la Théotokos Source de vie, le peintre est revenu au modèle du XIVe siècle qui faisait de la Vierge la Reine des lieux, comme l'exprime d'ailleurs la dédicace de ce Constantin dont nous ne savons rien...

Les icônes de ce type devait correspondre à une clientèle déjà fortunée qui choisissait (ou commandait) un sujet ou l'autre, et il y avait, évidemment des œuvres plus modestes comme il est d'usage dans les lieux de pèlerinage, sans compter les gravures. La force surnaturelle qui se manifestait sur les lieux de la Zoodochos Pege était transmise par l'icône ${ }^{28}$. Avec cette image Constantin emportait chez lui quelque chose du pouvoir thaumaturge de la Source, et ses ferventes prières à la Théotokos orante, présentée dans un cadre doré évoquant celui d'un autel ${ }^{29}$, le remettaient en condition.

Les images tardives de la Zoodochos Pege ont encore le mérite de nous restituer quelques choses de la religiosité byzantine à Constantinople à propos d'un lieu de pèlerinage remontant au Ve siècle.

Etampes, 8-12-1999
28. Ce travail mental qui fait de l'icône un objet thaumaturge porteur des vertus du sujet représenté est bien connu; citons A. Grabar, Martyrium, Recherches sur le culte des reliques et l'art chrétien antique, II, Paris 1946, p. 343-357 ( « Des reliques aux icônes » : pouvoir des icônes et particulièrement de celles de la Vierge).
29. On remarque les deux grands oiseaux qui surmontent le cadre, aussi bien sur l'icône Abou-Adal et un peu différents sur celle du Musée d'Athènes. On peut supposer une réduction de la décoration d'une iconostase du XVIIIe siècle (?) mais plus encore d'une composition baroque d'église d'Europe centrale. 